\title{
Transcription start sites at the end of protein-coding genes
}

\author{
Ming-Yu Huang and Ji-Long Liu* (D)
}

\begin{abstract}
Previous studies demonstrated that massive induction of transcriptional readthrough generates downstream of gene-containing transcripts (DoGs) in cells under stress condition. Here, we analyzed TSS-seq (transcription start site sequencing) data from the DBTSS database. We investigated TSS tags at the end of gene for all pan-stress and untreated-cell DoGs, in comparison with expression-matched non-DoGs. We observed significantly more TSS tags at the end of pan-stress and untreated-cell DoG genes than non-DoG genes, even though their TSS tags in the promoter is the same. Importantly, the median value of TSS tags at gene end normalized to gene promoter is significantly higher than the median expression ratio of short DoG to host gene and of long DoG to host gene. Our results indicate that downstream overlapping long non-coding RNAs derived from the TSS at the gene end may be an important source of DoGs.
\end{abstract}

Keywords: Transcriptional readthrough, Downstream of gene-containing transcripts (DoGs), TSS-seq

\section{Background}

Vilborg et al. analyzed nuclear transcriptome changes in SK-N-BE(2)C human neuroblastoma cells [1] and NIH3T3 mouse fibroblast cells [2] under heat shock, osmotic stress, and oxidative stress by using RNA-seq. They observed massive induction of transcriptional readthrough, or downstream of gene-containing transcripts (DoGs), under all stress conditions. Being long (often $>45 \mathrm{~kb}$ ) and diverse ( 2000 species), DoGs may contribute significantly to the transcriptome.

Previously, we have demonstrated that the progesterone receptor (PGR) gene processes a very long 3 '-UTR of approximately $10 \mathrm{~kb}$ and this length can be further extended in the monkey endometrium from the view of sequencing data [3]. However, we have found that this extension is not due to a readthough, but an independent transcription start site (TSS) at the end of PGR, resulting a sense long non-coding RNA (lncRNA) overlapping with PGR 3'-UTR. Thus, we questioned whether these DoGs observed by Vilborg et al. [1,2] are downstream overlapping lncRNAs instead of readthrough products from the promoter of protein-coding genes.

* Correspondence: jilongliu@scau.edu.cn

College of Veterinary Medicine, South China Agricultural University, Guangzhou, China

(c) The Author(s). 2018 Open Access This article is distributed under the terms of the Creative Commons Attribution 4.0 International License (http://creativecommons.org/licenses/by/4.0/), which permits unrestricted use, distribution, and reproduction in any medium, provided you give appropriate credit to the original author(s) and the source, provide a link to the Creative Commons license, and indicate if changes were made. The Creative Commons Public Domain Dedication waiver (http://creativecommons.org/publicdomain/zero/1.0/) applies to the data made available in this article, unless otherwise stated.
To answer this question, we performed a bioinformatic analysis of the public data. Our preliminary results challenge the readthough model proposed by Vilborg et al. $[1,2]$.

\section{Methods}

The TSS-seq data performed on NIH3T3 cells were downloaded from the DataBase of Transcriptional Start Sites (DBTSS, https://dbtss.hgc.jp). The DNaseI data for NIH3T3 cells as well as Pol2, H3K4m1, and H3K4m3 for MEF (mouse embryo fibroblast) cells were derived from the ENCODE project (https://www.encodeproject.org). The UCSC Genome Browser (http://genome.ucsc.edu/) was used to display TSS-seq data and chromatin features for four representative DoGs: Hnrnpa2b1, Txn1, Hspa8, and Ifitm2. The genomic coordinates were based on mouse $\mathrm{mm} 9$ genome assembly.

In addition to the four representative DoGs, we extracted the genomic coordinates for all the DoGs described by Vilborg et al. [2]. The number of TSS tags at 1-kb region of a gene promoter and gene end were summarized according to TSS-seq data. Because DoGs and non-DoGs differ in size and gene expression levels, we constructed an equal size expression-matched subset for non-DoGs by randomly sampling using in-house PERL scripts. Difference between groups was tested by the nonparametric Mann-Whitney $U$ test implemented in MATLAB (MathWorks, version 7.5). 

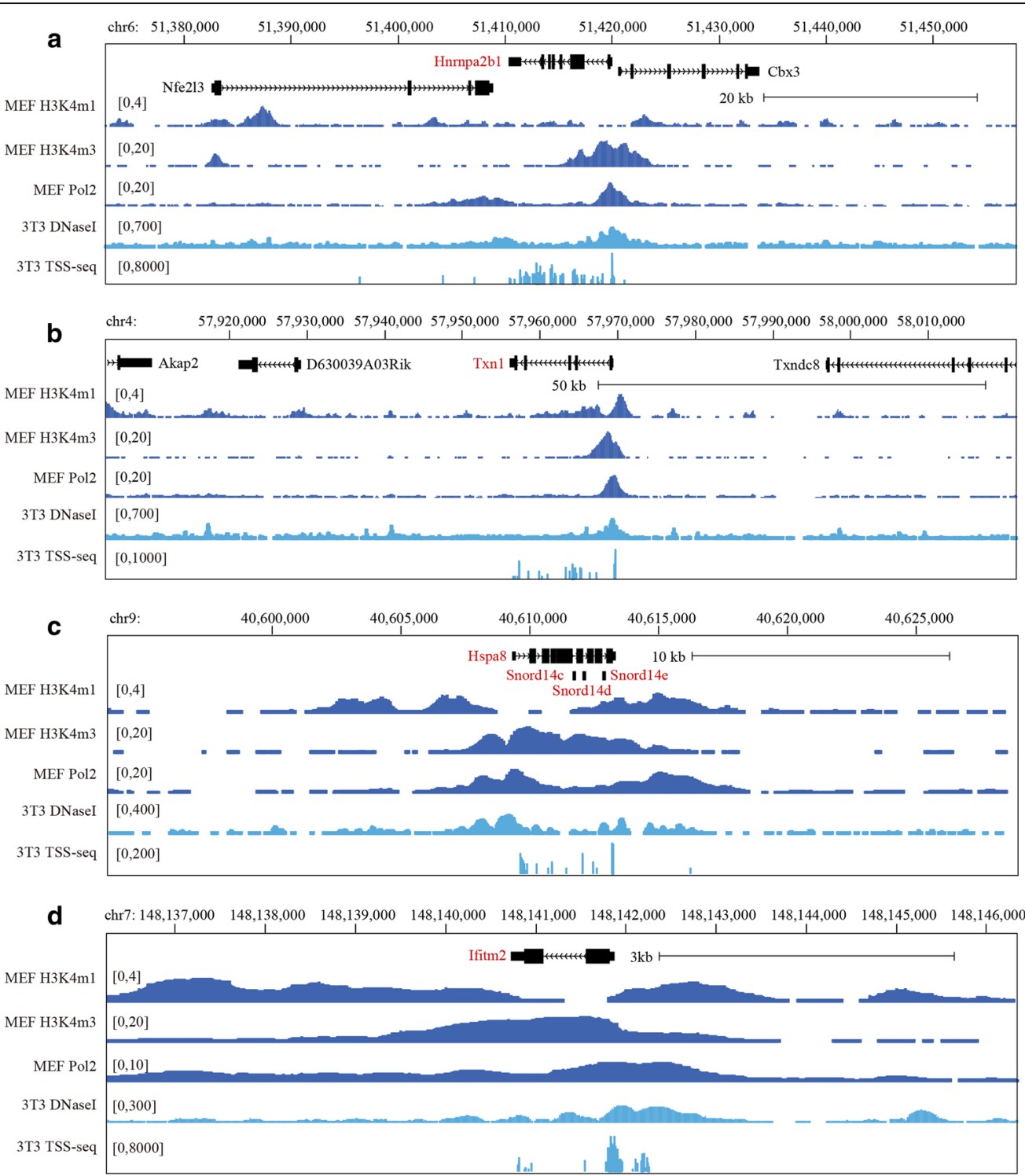

Fig. 1 TSS-seq data and chromatin features for four representative DoGs. a Hnrnpa2b1. b Txn1. c Hspa8. d Ifitm2. Open chromatin in the genome is marked by Pol2 and DNasel occupancy. H3K4me3 is a promoter marker and H3K4me1 is an enhancer marker

Table 1 Statistical analysis of TSSs at gene end

\begin{tabular}{|c|c|c|c|}
\hline Category & Type & Median (25th-75th quantiles) & $P$ value \\
\hline \multirow[t]{3}{*}{ Promoter-1 k } & Non-DoGs & $204(61-639)$ & \\
\hline & Pan-stress DoGs & $207(47-782)$ & 0.687 \\
\hline & Untreated DoGs & $203(78-718)$ & 0.534 \\
\hline \multirow[t]{3}{*}{ End-of-gene-1 k } & Non-DoGs & $15(2-51)$ & \\
\hline & Pan-stress DoGs & $22(5-75)$ & $0.0000285^{*}$ \\
\hline & Untreated DoGs & $23(6-79)$ & $0.00000276^{*}$ \\
\hline \multirow[t]{3}{*}{ End-of-gene-1 kb/promoter-1 kb } & Non-DoGs & $0.0769(0.0138-0.4071)$ & \\
\hline & Pan-stress DoGs & $0.1149(0.0193-0.6251)$ & $0.000289^{*}$ \\
\hline & Untreated DoGs & $0.1279(0.0191-0.7435)$ & $0.0000193^{*}$ \\
\hline
\end{tabular}

Expression-matched non-DoGs were randomly selected, and Mann-Whitney $U$ test was performed. $P$ values were calculated by comparing to non-DoGs $* P<0.05$ 


\section{Results and discussion}

By combining oligo-capping with high throughput sequencing, the TSS-seq approach is able to collect genome-wide TSS information together with a quantitative analysis of the expression levels of transcripts [4]. We examined TSS-seq data performed on NIH3T3 cells from the DBTSS database [5]. For all four representative DoGs (Hnrnpa2b1, Txn1, Hspa8, and Ifitm2) [2], the number of TSS tags at the end of a gene is one order of magnitude lower than that at a promoter, except Hspa8 (Fig. 1). Hspa8 exhibits higher number of TSS tags at the gene end compared to the promoter, likely due to intronic snoRNAs. These TSSs may generate lncRNAs with an independent promoter at the gene end.

We next investigated TSS tags at the end of a gene for all pan-stress and untreated-cell DoGs, in comparison with expression-matched non-DoGs. We observed significantly more TSS tags at the end of pan-stress and untreated-cell DoG genes than those of non-DoG genes, even though their TSS tags in the promoter is the same. Furthermore, we normalized the number of TSS tags at the gene end to the number of TSS tags at the promoter of the same gene. Significance was also reached for the normalized data (Table 1 and Additional file 1: Figure S1).

Additionally, the median value of TSS tags at gene end normalized to gene promoter is 0.1088 , slightly higher than the median expression ratio of short DoG to host gene (0.0146) and of long DoG to host gene (0.0067). These results indicate that TSSs at a gene end may be an important source of DoGs.

\section{Conclusion}

Taken together, by analyzing TSS-seq data, we suggested that TSSs at the gene end may be an important major source of DoGs. Therefore, TSS-seq along with a large scale of Northern blot and tiling PCR experiments are required by Vilborg et al. $[1,2]$ to support their idea that most DoGs are continuous transcripts caused by a readthrough of protein-coding genes.

\section{Additional file}

Additional file 1: Figure S1. Statistical analysis of TSSs at gene end (related to Table 1). (A) Number of TSS tags at 1-kb region of gene promoter and gene end, among pan-stress DoGs, untreated-cell DoGs, and non-DoGs. (B) Normalized number of TSS tags at gene end to the number of TSS tags at gene promoter, among pan-stress DoGs, untreated-cell DoGs, and non-DoGs. (TIFF $468 \mathrm{~kb})$

\section{Abbreviations}

DoGs: Downstream of gene-containing transcripts; Hnrnpa2b1: Heterogeneous nuclear ribonucleoprotein a2/b1; Hspa8: Heat shock 70 kDa protein 8; Ifitm2: Interferon-induced transmembrane protein 1; IncRNA: Long non-coding RNA; PGR: Progesterone receptor; TSS: Transcription start site; Txn1: Thioredoxin 1

Acknowledgements

Not applicable.

\section{Funding}

This work was funded by the National Natural Science Foundation of China (grant numbers 31771665 and 31271602 to Ji-Long Liu).

Availability of data and materials

Please contact the author for data requests.

Authors' contributions

$J L L$ designed/performed the research and wrote the paper. MYH analyzed the data. Both authors read and approved the final manuscript.

Ethics approval and consent to participate

Not applicable.

Consent for publication

Not applicable.

Competing interests

The authors declare that they have no competing interests.

\section{Publisher's Note}

Springer Nature remains neutral with regard to jurisdictional claims in published maps and institutional affiliations.

Received: 18 February 2018 Accepted: 9 March 2018

Published online: 16 March 2018

\section{References}

1. Vilborg A, Passarelli MC, Yario TA, Tycowski KT, Steitz JA. Widespread inducible transcription downstream of human genes. Mol Cell. 2015;59(3):449-61.

2. Vilborg A, Sabath N, Wiesel Y, Nathans J, Levy-Adam F, Yario TA, Steitz JA, Shalgi R. Comparative analysis reveals genomic features of stress-induced transcriptional readthrough. Proc Natl Acad Sci USA. 2017;114(40):E8362E8371.

3. Liu JL, Liang XH, Su RW, Lei W, Jia B, Feng XH, Li ZX, Yang ZM. Combined analysis of microRNome and $3^{\prime}$-UTRome reveals a species-specific regulation of progesterone receptor expression in the endometrium of rhesus monkey. J Biol Chem. 2012;287(17):13899-910.

4. Tsuchihara K, Suzuki Y, Wakaguri H, Irie T, Tanimoto K, Hashimoto S, Matsushima K, Mizushima-Sugano J, Yamashita R, Nakai K, et al. Massive transcriptional start site analysis of human genes in hypoxia cells. Nucleic Acids Res. 2009;37(7):2249-63.

5. Suzuki A, Wakaguri H, Yamashita R, Kawano S, Tsuchihara K, Sugano S, Suzuki Y, Nakai K. DBTSS as an integrative platform for transcriptome, epigenome and genome sequence variation data. Nucleic Acids Res. 2015;43(Database issue):D87-91.

Submit your next manuscript to BioMed Central and we will help you at every step:

- We accept pre-submission inquiries

- Our selector tool helps you to find the most relevant journal

- We provide round the clock customer support

- Convenient online submission

- Thorough peer review

- Inclusion in PubMed and all major indexing services

- Maximum visibility for your research 